\title{
Multifocal papillary thyroid cancer in children and adolescents: 12-year experience in a single center
}

\author{
Jiaying Chen ${ }^{1,2 \#}$, Naisi Huang ${ }^{1,2 *}$, Qinhai $\mathrm{Ji}^{1,2}$, Yu Wang ${ }^{1,2}$, Yongxue Zhu ${ }^{1,2}$, Duanshu $\mathrm{Li}^{1,2}$ \\ ${ }^{1}$ Department of Head and Neck Surgery, Furan University Shanghai Cancer Center, Shanghai 200032, China; ${ }^{2}$ Department of Oncology, Shanghai \\ Medical College, Fudan University, Shanghai 200032, China \\ Contributions: (I) Conception and design: J Chen, N Huang; (II) Administrative support: Y Wang, Q Ji, Y Zhu, D Li; (III) Provision of study \\ materials or patients: Y Wang, Q Ji, Y Zhu, D Li; (IV) Collection and assembly of data: J Chen, Y Wang, Q Ji, Y Zhu, D Li; (V) Data analysis and \\ interpretation: J Chen, N Huang; (VI) Manuscript writing: All authors; (VII) Final approval of manuscript: All authors. \\ "These authors contributed equally to this work. \\ Correspondence to: Qinhai Ji. Department of Head and Neck Surgery, Furan University Shanghai Cancer Center, 270 Dong An Road, Shanghai \\ 200032, China. Email: chen_lain@hotmail.com.
}

Background: Thyroid cancer is the most common endocrine malignant disease in children and adolescents. There is a trend of more conservative strategies including lobectomy and less radioactive iodine therapy (RAI) in multifocal papillary thyroid cancer (PTC) for its good survival outcome. The aim of our study was to define long-time outcome of a large cohort of multifocal PTC patients less than 20 years old treated at our institution.

Methods: Data were collected from 276 cases who were initially diagnosis of PTC under the age of 20 from January 2006 to December 2015 at Fudan University Shanghai Cancer Center. All patients received total/near total thyroidectomy or lobectomy. Therapeutic central-compartment (level VI) and lateral neck lymph node dissection performed for patients with clinically involved neck nodes. RAI therapy used in selected patients. No patients received chemotherapy or kinase inhibitor therapy. Thyroid-stimulating hormone (TSH) suppression therapy was performed in all patients for at least 5 years.

Results: Ninety among 276 were multifocal PTC patients and included in this study. The median followup time was 54.28 months, ranging from 6.10 to 141.27 months. Fifteen patients had tumor recurrence during the follow-up. On Kaplan-Meier survival curves, lymphovascular invasion and extrathyroidal extension was associated with a decline in recurrence-free survival. However, there was no difference in recurrence-free survival curves in patients no matter which treatment they had received, either lobectomy or total thyroidectomy, RAI or not.

Conclusions: More conservative strategies including lobectomy and less RAI in multifocal PTC among children and adolescents are safe and effective.

Keywords: Papillary thyroid cancer (PTC); multifocal; pediatric and adolescents; lobectomy; radioactive therapy; prognosis

Submitted May 21, 2019. Accepted for publication Aug 28, 2019.

doi: 10.21037 /gs.2019.09.03

View this article at: http://dx.doi.org/10.21037/gs.2019.09.03 


\section{Introduction}

With the incidence increasing every year, approximately 90,000 new cases of thyroid cancer were diagnosed in China every year (1). Among people aged from 15 to 19 years, thyroid cancer is the most common endocrine malignant disease, accounting for $11 \%$ of all pediatric cancers (2). However, pediatric papillary thyroid cancer (PTC) is still a rare disease. Due to the lack of clinical trials, treatment options remain controversial. When compared to adult patients, children present with a more multifocal disease at the time of diagnosis, which is recognized as a high-risk factor for the progression of PTC (3), thereby requiring the application of more aggressive treatment. Recently, there has emerged a trend of more conservative strategies including lobectomy and less radioactive iodine therapy (RAI) in multifocal PTC due to its good survival outcome (4). A multi-disciplinary therapy for multifocal pediatric PTC is advocated, but no clinical guidelines have yet been fully established. In our center, not all multifocal pedantic patients receive total thyroidectomy (TT). Thus, RAI therapy would not be routinely used for those patients. Previous reports regarding pediatric PTC are mostly singleinstitution and based on local clinical experience (5-9). Until now, there has been no relevant data in Chinese multifocal pediatric PTC populations reported.

Our study aimed to define the long-term outcomes of a large cohort of multifocal PTC patients less than 20 years old treated at our institution. We hoped to investigate the performance of the surgical-based therapy used for the treatment of multifocal pediatric PTC and to determine whether it is appropriate to treat with a more conservative strategy to minimize the side effects in children and adolescents.

\section{Methods}

\section{Patients}

Data was collected from 276 cases who were initially diagnosed with PTC under the age of 20 from January 2006 to December 2015 at the Fudan University Shanghai Cancer Center. Patients included in this study needed to meet the following criteria: (I) histologic confirmation of PTC; (II) no aggressive variant type of PTC; (III) more than two tumor foci; (IV) under the age of 20 at diagnosis; and $(\mathrm{V})$ with informed consent.

All the pathological results were reconfirmed by two pathologists in the head and neck department according to the World Health Organization classification (10). Histopathologic variants of PTC that were associated with more unfavorable outcomes such as tall cell, columnar cell, and hobnail variants of PTC were excluded in this study. The protocol was approved by the Ethics Committee of Fudan University Shanghai Cancer Center.

The tumor stage was classified according to the American Joint Committee on Cancer TNM (AJCC/ TNM) staging system, 7th edition. All patients participated in this study received total/near TT or lobectomy by highvolume thyroid surgeons (Q Ji performed 30 cases; Y Wang performed 24 cases; Y Zhu performed 22 cases; D $\mathrm{Li}$ performed 14 cases). Patients would have lobectomy as opposed to TT if they met all of the following conditions: (I) unilateral disease; (II) primary tumor size $<4 \mathrm{~cm}$; (III) without extrathyroidal extension and lymphovascular invasion; and (IV) no clinical suspect lymph nodes in the lateral neck. Therapeutic central-compartment (level VI) and lateral neck lymph node dissection were performed for patients with clinically involved neck nodes with or without biopsy-proven.

RAI therapy was used in the selected patients. Patients were recommended to have RAI after TT if they met any of the following conditions: (I) lymphovascular invasion; (II) extrathyroidal extension; (III) more than 14 metastatic lymph nodes (no matter the size); (IV) primary tumor size $>4 \mathrm{~cm}$; (V) any metastatic lymph nodes $\geq 3 \mathrm{~cm}$; and (VII) distant metastasis. No patients received chemotherapy or kinase inhibitor therapy. Thyroid stimulating hormone suppression therapy was performed in all patients for at least 5 years.

\section{Follow-up and clinical outcomes}

Patients were reviewed in the clinic every 3 months in the first year and every 6 months for the next 4 years, then annually after 5 years. During the follow-up, laboratory tests, ultrasound of the neck, and pulmonary computed tomography (CT) were arranged for the patients. Ultrasound on the contralateral lobe was also arranged for patients who received lobectomy only. When the suspected lymph nodes or nodule in the neck area occurred, a fineneedle biopsy was used to confirm metastasis. Patients who received TT and RAI were also monitored by thyroglobulin $(\mathrm{Tg})$ and a diagnostic ${ }^{131} \mathrm{I}$ whole body scan during the follow-up. The recurrent event and clinical status at the time of the last follow-up was recorded for each patient. During the follow-up, the recurrent site was neck lymph 
nodes for all of the local recurrent patients. The lung and bone were two sites of distant metastasis. None of the patients had a persistent elevated $\mathrm{Tg}$ level without a structural disease. At the last follow-up time, the status of patients was recorded as follows: (I) no evidence of disease: no biochemical or structural evidence of disease; (II) progressive disease: tumor developed progressively in the neck or distant area; (III) stable disease: tumor developed in the neck or distant area, but the disease was in a stable status.

\section{Statistical analysis}

Continuous variables were expressed as the mean and standard deviation (SD), and categorical variables were expressed as number and frequencies (\%). The Student t-test, Wilcoxon rank-sum test, Pearson Chi-square test, and Fisher exact test were used to compare continuous and categorical variables according to the type and distribution of data.

The log-rank test was used to compare the recurrencefree survival rate of different subgroups. Cox proportional hazard regression models were used to estimate the hazard ratio (HR) and $95 \%$ CI for incident recurrence.

All of the $\mathrm{P}$ values and $95 \%$ CIs were estimated in a two-tailed manner. Differences were considered to be statistically significant at $\mathrm{P}<0.05$. Data were analyzed using SAS 9.4 (SAS Institute Inc., Cary, NC, USA).

\section{Results}

\section{General characteristics of the study population}

Ninety-four among 276 were multifocal PTC patients in which 1 case was lost in the follow-up, and another 3 cases were diagnosed of tall cell variant of PTC. Finally, 90 cases were included in this study cohort. The clinicopathologic characteristics of the 90 patients included in this study are provided in Table 1. The median age at initial diagnosis was 16 , ranging from 8 to 19 . The majority of patients were female (69 cases, $76.67 \%$ ). The size of largest dominant tumor in more than half of the patients $(64.44 \%)$ was $>2 \mathrm{~cm}$ while the most non-dominant tumor was $<2 \mathrm{~cm}$. Extrathyroidal extension were present in 44 cases. Lymphovascular invasion were present in 16 patients. Most multifocal PTC patients in the study were bilateral in the cancer side with a median of 3.12 foci. Seventynine of 90 cases received total/near TT, and the others received lobectomy only. Eleven of 15 cases with unilateral multifocal PTC received lobectomy only. Prophylactic neck dissections were not performed in any of the patients. More than $80 \%$ of all the patients received therapeutic level VI and/or lateral neck dissections. Eighty-eight patients involved in the study had nodal involvement (97.78\%), with 14 patients with $\mathrm{N} 1 \mathrm{a}$ disease and 74 patients with $\mathrm{N} 1 \mathrm{~b}$ diseases. Five cases were found to have lung metastasis at the time of diagnosis. Only 47 patients had RAI after surgery including 5 cases who had lung metastasis at the time of diagnosis ( $M 1$ cases).

\section{Clinical outcomes of the cohort}

The median follow-up time was 54.28 months, ranging from 6.10 to 141.27 months. Fifteen patients had tumor recurrence during the follow-up time. Most of them were female, and all of them had lymph node involvement in the lateral neck. Extrathyroidal extension developed in 10 cases and lymphovascular invasion developed in 7 cases. Four of the 15 recurrent patients received RAI after initial therapy. Among these 15 patients, 1 patient was in the M1 stage at the time of diagnosis. Her metastatic pulmonary foci were stable after initial RAI therapy. After 21.6 months, we found recurrent lymph nodes in her neck area. Supplementary neck dissection was performed successfully, and the patient had stable disease (lung) by the end of the follow-up. Another 6 patients who only had neck recurrence were all treated with supplementary neck dissection and achieved no evidence of disease by the end of follow-up. Three cases developed pulmonary metastasis after long-term follow-up. The recurrence time was 67.96, 77.04, and 110.04 months. After RAI therapy, 2 failed in the therapy, and only 1 had a stable disease by the end of the follow-up. Two cases developed recurrent disease in the neck and lung at the same time. In 3 cases, there were first found recurrent lymph nodes in the neck, and pulmonary metastasis developed 2 years later. All 5 of these patients did not have a stable outcome after therapy. Among the other 4 patients who were initially diagnosed with $\mathrm{M} 1$ stage, one had no evidence of disease, and 4 had stable disease at the last follow-up. In conclusion, at the last follow-up time, 78 patients had no evidence of disease, 7 patients had progressive disease, and 5 had stable disease (Table 2).

\section{Risk factors of recurrence}

The univariate Cox regression analysis showed that age, 
Table 1 The clinicopathologic characteristics of the 90 patients

\begin{tabular}{|c|c|}
\hline Characteristics & Value \\
\hline \multicolumn{2}{|l|}{ Age at diagnosis, years } \\
\hline Mean \pm SD & $14.61 \pm 2.84$ \\
\hline Median & 15 \\
\hline Range & $8-19$ \\
\hline Female gender, n (\%) & $69(76.67)$ \\
\hline \multicolumn{2}{|c|}{ Size of largest dominant tumor, $\mathrm{n}(\%)$} \\
\hline$\leq 1 \mathrm{~cm}$ & $15(18.89)$ \\
\hline$>1, \leq 2 \mathrm{~cm}$ & $15(16.67)$ \\
\hline$>2, \leq 4 \mathrm{~cm}$ & $33(34.44)$ \\
\hline$>4 \mathrm{~cm}$ & $27(30.00)$ \\
\hline \multicolumn{2}{|c|}{ Size of non-dominant tumor, n (\%) } \\
\hline$\leq 1 \mathrm{~cm}$ & $73(81.11)$ \\
\hline$>1, \leq 2 \mathrm{~cm}$ & $11(12.22)$ \\
\hline$>2, \leq 4 \mathrm{~cm}$ & $5(5.56)$ \\
\hline$>4 \mathrm{~cm}$ & $1(1.11)$ \\
\hline \multicolumn{2}{|l|}{$\mathrm{N}$ stage, $\mathrm{n}(\%)$} \\
\hline N1A & $14(15.56)$ \\
\hline N1B & $74(82.22)$ \\
\hline \multicolumn{2}{|l|}{ M stage, n (\%) } \\
\hline M0 (stage I) & $85(94.44)$ \\
\hline M1 (stage II) & $5(5.56)$ \\
\hline ETE, n (\%) & $44(48.89)$ \\
\hline LVI, n (\%) & $16(17.78)$ \\
\hline \multicolumn{2}{|l|}{ Foci number, n (\%) } \\
\hline 2 & 47 (52.22) \\
\hline 3 & $13(14.44)$ \\
\hline 4 & $16(17.78)$ \\
\hline$>4$ & $14(15.56)$ \\
\hline \multicolumn{2}{|l|}{ Cancer side, n (\%) } \\
\hline Unilateral & 15 (16.67) \\
\hline Bilateral & 75 (83.33) \\
\hline \multicolumn{2}{|l|}{ Surgery, n (\%) } \\
\hline Lobectomy & 11 (12.22) \\
\hline Total/near total & 79 (87.78) \\
\hline Level VI & 79 (87.78) \\
\hline
\end{tabular}

Table 1 (continued)
Table 1 (continued)

\begin{tabular}{lc}
\hline Characteristics & Value \\
\hline Lateral neck & $77(85.56)$ \\
Total LN harvested & \\
Harvested $\leq 37$ & $37(41.11)$ \\
$37<$ harvested $\leq 52$ & $17(18.89)$ \\
$52<$ harvested $\leq 68$ & $20(22.22)$ \\
Harvested $>68$ & $16(17.78)$ \\
Total LN involved & \\
Involved $\leq 14$ & $35(38.89)$ \\
$14<$ involved $\leq 20$ & $20(22.22)$ \\
$20<$ involved $\leq 27$ & $17(18.89)$ \\
Involved $>27$ & $18(20.00)$ \\
LN ratio & \\
Ratio $\leq 2$ & $33(36.67)$ \\
$2<$ ratio $\leq 3$ & $34(37.78)$ \\
Ratio $>3$ & $23(25.56)$ \\
RAI after surgery, $\mathrm{n}(\%)$ & \\
Yes & $47(52.22)$ \\
No & $437.78)$ \\
\hline SD, standard deviation; & \\
\hline
\end{tabular}

SD, standard deviation; LVI, lymphovascular invasion; ETE, extrathyroidal extension; LN, lymph node.

Table 2 Follow-up clinical outcomes of all of the patients $(n=90)$

\begin{tabular}{lc}
\hline Variables & Value \\
\hline Time from diagnosis to the last follow-up, months & \\
Mean \pm SD & $62.36 \pm 32.67$ \\
Median & 54.28 \\
Range & $6.10-141.27$ \\
Recurrence during the course of follow-up & 15 \\
Clinical outcome at the end of the follow up, $\mathrm{n}(\%)$ & \\
No evidence of disease & 78 \\
Recurrent disease(lung) & 7 \\
Persistent disease & 5 \\
\hline
\end{tabular}


Table 3 Univariable Cox analysis of factors associated with recurrence-free survival (local/distant)

\begin{tabular}{|c|c|c|}
\hline Characteristics & $\mathrm{HR}(95 \% \mathrm{Cl})$ & $P$ value \\
\hline Age & $0.82(0.69,0.96)$ & 0.0134 \\
\hline Sex (female) & $1.03(0.33,3.25)$ & 0.9583 \\
\hline \multicolumn{3}{|c|}{ Size of largest dominant tumor } \\
\hline$\leq 1 \mathrm{~cm}$ & References & - \\
\hline$>1, \leq 2 \mathrm{~cm}$ & $1.33(0.64,2.74)$ & 0.4448 \\
\hline$>2, \leq 4 \mathrm{~cm}$ & $0.82(0.44,1.53)$ & 0.5359 \\
\hline$>4 \mathrm{~cm}$ & $0.98(0.51,1.87)$ & 0.9496 \\
\hline \multicolumn{3}{|c|}{ Size of non-dominant tumor } \\
\hline$\leq 1 \mathrm{~cm}$ & Reference & - \\
\hline$>1, \leq 2 \mathrm{~cm}$ & $2.64(0.81,8.43)$ & 0.1077 \\
\hline$>2, \leq 4 \mathrm{~cm}$ & - & - \\
\hline$>4 \mathrm{~cm}$ & $2.84(0.33,24.27)$ & 0.3396 \\
\hline M stage & $1.09(0.14,8.45)$ & 0.9346 \\
\hline LVI & $3.15(1.07,9.25)$ & 0.0366 \\
\hline ETE & $2.67(0.85,8.45)$ & 0.0439 \\
\hline \multicolumn{3}{|l|}{ Foci number } \\
\hline$\leq 2$ & Reference & - \\
\hline 3 & $1.21(0.32,4.58)$ & 0.7784 \\
\hline 4 & $1.14(0.30,4.38)$ & 0.8501 \\
\hline$>4$ & $0.50(0.06,4.05)$ & 0.5141 \\
\hline Cancer side (unilateral) & $0.67(0.21,2.17)$ & 0.5033 \\
\hline
\end{tabular}

Table 3 (continued)

extrathyroidal extension and lymphovascular invasion were the risk factors for postoperative recurrence (Table 3); however, on multivariate adjustment, the lymphovascular invasion was the only independent risk factor for recurrence (Table 4). On Kaplan-Meier survival curves of patients with PTC, lymphovascular invasion and extrathyroidal extension were associated with a decline in recurrencefree survival (Figures 1,2). In a striking contrast, there was no difference in the recurrence-free survival curves in patients, irrespective of the treatment they received whether lobectomy or TT, RAI or no RAI (Figures 3,4).

\section{Discussion}

To our knowledge, this is the first study to define the long-
Table 3 (continued)

\begin{tabular}{lcc}
\hline Characteristics & HR $(95 \% \mathrm{Cl})$ & $\mathrm{P}$ value \\
\hline Surgery & & \\
Lobectomy & Reference & - \\
Total/near total & $1.43(0.32,6.35)$ & 0.6397 \\
Level VI & $0.68(0.09,5.25)$ & 0.7110 \\
Lateral neck & - & - \\
Total LN involved & & \\
Involved $\leq 14$ & References & - \\
14<involved $\leq 20$ & $1.48(0.30,6.65)$ & 0.6655 \\
$20<$ involved $\leq 27$ & $2.52(0.60,10.47)$ & 0.2049 \\
$27<$ involved & $1.91(0.47,7.69)$ & 0.3637 \\
Total LN involved & & \\
Harvested $\leq 37$ & References & - \\
$37<$ harvested $\leq 52$ & $0.72(0.14,3.75)$ & 0.6992 \\
$52<$ harvested $\leq 68$ & $1.12(0.26,4.75)$ & 0.8766 \\
Harvested $>68$ & $1.98(0.57,6.86)$ & 0.2799 \\
LN ratio & & \\
Ratio $\leq 2$ & Reference & - \\
$2<$ ratio $\leq 3$ & $1.82(0.59,5.69)$ & 0.3004 \\
Ratio $>3$ & $0.60(0.12,3.13)$ & 0.5457 \\
RAl after surgery & $0.78(0.25,2.44)$ & 0.6695 \\
\hline Cl, confidence interval; ETE, extrathyroidal extension; LVI, \\
lymphovascular invasion; LN, lymph node; RAl, radioactive \\
ablation. & & \\
& &
\end{tabular}

time outcomes of multifocal PTC patients in Chinese children and adolescents with a median follow-up for 5 years. Although most patients have N1 disease, the recurrence rate was low (15/90) in this cohort. At the last follow-up time, 78 patients had no evidence of disease and no one died from thyroid cancer. The results indicating the low-risk behavior and proper treatment of this presentation.

Multifocal involvement is a common presentation in PTC of children and adolescents. According to our data, 1 in 3 pediatric PTC patients had multifocal disease. Tumor multifocality is often regarded as a risk factor for the poor prognosis of PTC, prompting more aggressive treatments. However, there is no well-established evidence to support this practice. Previous studies have controversial opinions about whether multifocality was a risk factor for thyroid 
Table 4 Hazard ratios of the clinicopathological characteristics for recurrence of thyroid cancer in multivariate Cox proportional hazards analysis

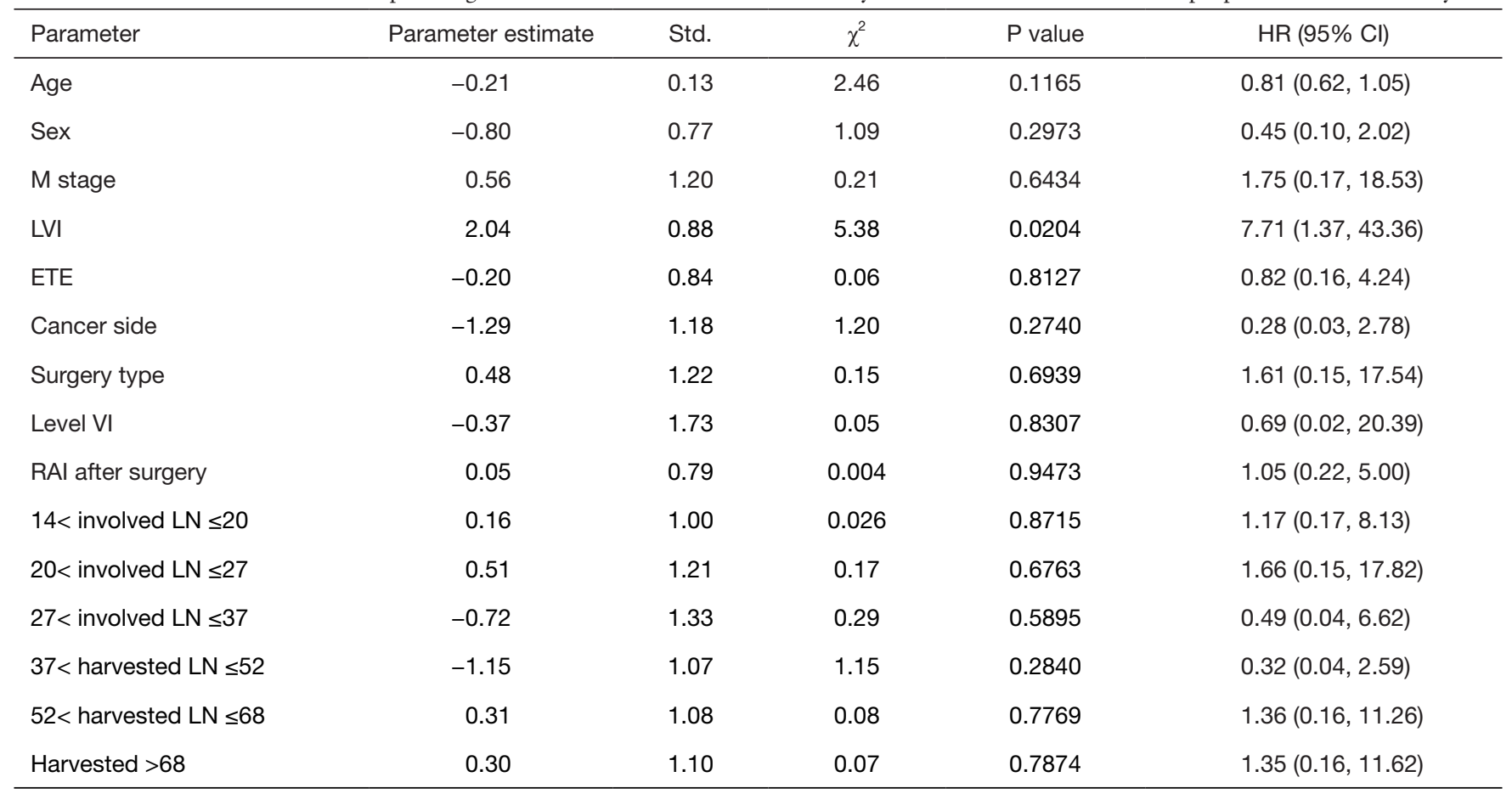

ETE, extrathyroidal extension; LVI, lymphovascular invasion; LN, lymph node; RAI, radioactive ablation; HR, hazard ratio; Cl, confidence interval.

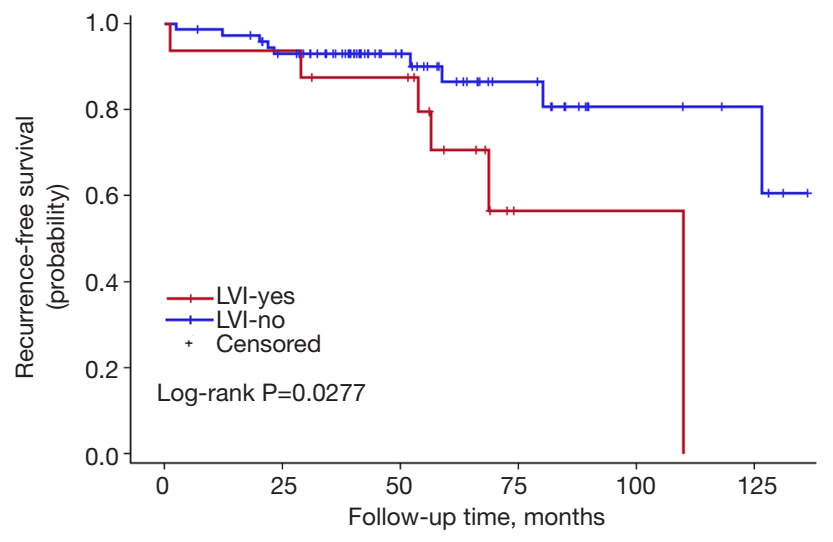

Figure 1 Kaplan-Meier survival curve analysis of the effect of lymphovascular invasion on disease recurrence-free survival $(\mathrm{P}=0.0277)$.

cancer recurrence (11-16). Recently, a multicenter study of a large cohort demonstrated that tumor multifocality has no independent risk prognostic value in clinical outcomes of PTC (17). Thus, its indiscriminative use as an independent risk factor prompting overtreatment of patients should be avoided. In the 2015 update of the American Thyroid

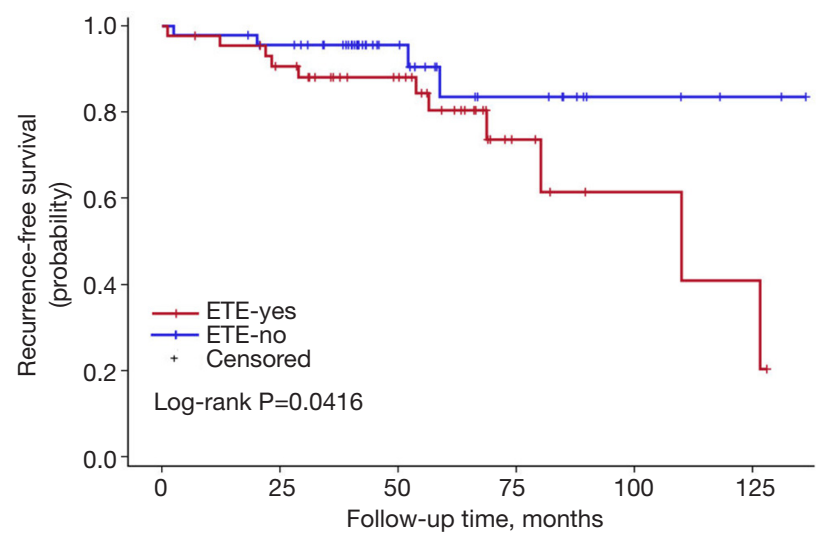

Figure 2 Kaplan-Meier survival curve analysis of extrathyroidal extension on disease recurrence-free survival $(\mathrm{P}=0.0416)$.

Association guidelines, multifocality itself was not regarded as a risk factor in the three-tiered categorical risk of recurrence system (4).

Compared to adults, children present with more lymph nodes involved and lung metastases. The rate of lymph node metastasis is reported to be about $60-80 \%$ and the rate 


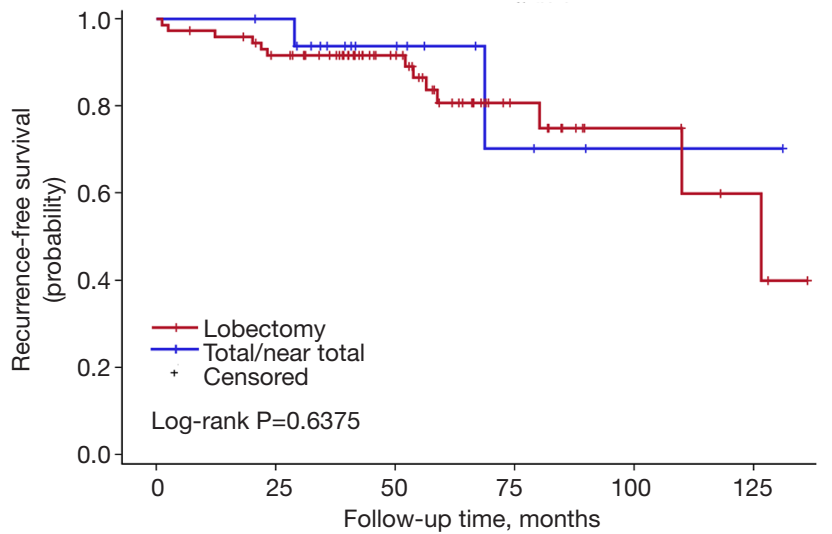

Figure 3 Kaplan-Meier survival curve analysis of the surgery type on disease recurrence-free survival $(\mathrm{P}=0.6375)$.

of lung metastasis is around $10 \%(18,19)$. In our study, 88 patients had nodal involvement (97.78\%), with 14 patients with N1a disease and 74 patients with N1b disease. Five of 90 cases presented with lung metastases at initial diagnosis. However, their biological effects were moderate, progression was slow, and the final prognosis was good after active treatment. Pediatric PTC is a rare disease. Most treatments for pediatric PTC were learned from experience in the adult PTC population. The latest ATA guidelines advocate for a more conservative treatment including lobectomy and less RAI in low-risk PTC patients. Paradoxically, ATA guidelines for pediatric thyroid cancer did not update its views: for the majority of patients with PTC, TT is recommended as an initial surgical approach (20). This recommendation for more comprehensive thyroid surgery in pediatric patients is based on multiple data showing an increased incidence of bilateral and multifocal disease (30\% and $65 \%$, respectively) (21-23). However, in the case that multifocality is not regarded as a risk factor, TT or near-total TT may not be the only choice for the multifocal patients. Pediatric thyroid cancer patients may be at a low risk for death but a higher risk for long-term harm from overly aggressive treatment. The complication of TT includes permanent hypocalcemia and vocal paralysis, which may affect children's growth and development, even threatening life. Under these circumstances, we advise that lobectomy and no RAI could be one of the treatment choices for the selective multifocal patients.

Our data indicated the possibility of a more conservative treatment in this real-world treatment pattern. On KaplanMeier survival curves of patients with PTC, lymphovascular invasion and extrathyroidal extension were associated with

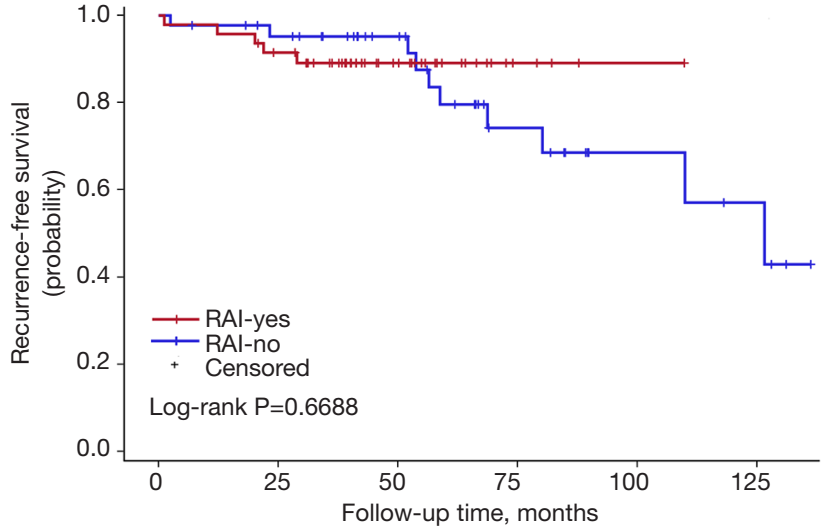

Figure 4 Kaplan-Meier survival curve analysis of RAI on disease recurrence-free survival $(\mathrm{P}=0.6688)$.

a decline in recurrence-free survival. In a striking contrast, there was no difference in recurrence-free survival curves in patients regardless of whether they received lobectomy or TT, RAI or no RAI. For those with ipsilateral multifocal foci and minor lymph nodes without lymphovascular invasion and extrathyroidal extension, lobectomy may be a proper treatment due to its low recurrence risk. In our study, 11 patients received lobectomy alone. During the follow-up, 2 of them had recurrent disease on the lateral neck. Both of them were treated with supplementary neck dissection and achieved no evidence of disease by the end of follow-up. To our knowledge although recurrent disease occurred in those 2 patients, until the last follow-up, they were free of disease. The trend toward a worse outcome with lobectomy may occur due to the relatively small cases of the population in the lobectomy group (11 vs. 79). The important fact is that the Kaplan-Meier survival curves did not show a significant difference between the two populations with different surgical types.

The goal of RAI is to decrease the risks of thyroid cancer recurrence and theoretically to improve mortality by eliminating iodine-avid disease. RAI was proposed as a specific treatment for DTC in 1946 and has been broadly applied for both adults and children (24). Universal administration of RAI for pediatric patients has not been accepted due to the conflicting results regarding the potential benefit from RAI. In one large study, RAI did not decrease the risk for loco-regional recurrence, distant metastases, or all-sites recurrence compared with surgery alone, but there was a trend toward reduction in the risk of distant metastases $(\mathrm{P}=0.06)$ (7). Unfortunately, the overall survival (OS) was reduced in patients who received ${ }^{131} \mathrm{I}$, 
and the chance of second malignancies was also increased. Another study also found no improvement in disease-free survival (DFS) following adjuvant RAI (25), but additional studies revealed a significant improvement in DFS for children with PTC treated with ${ }^{131} \mathrm{I}$ and no clear increase in the risk of second primary malignancies (26). Until now, no published data has confirmed the survival benefit of RAI for children. Furthermore, with the increased awareness of the potential long-term side effects of RAI, there has been growing opinion that RAI may not be necessary for patients who have a low likelihood of benefit from therapy. The majority of available data are based on a non-stratified approach in which all children underwent TT and variable extent of lymph node dissection, and the majority received therapeutic ${ }^{131}$ I. So the utility of lobectomy and no RAI have not fully validated. In our retrospective study, only 47 of 90 patients had RAI after surgery including 5 cases in whom lung metastasis was found at the time of diagnosis (M1 cases). For us, the challenge is to reduce the unnecessary exposure of ${ }^{131} \mathrm{I}$ to children who may not benefit without increasing disease-specific morbidity and mortality. The decision of RAI treatment was based both on the doctor's advice and the patients' wishes in the realworld treatment pattern. There were 5 patients who met the conditions but refused to receive RAI in our study. Two were concerned that the exposure of the radioactive iodine might lead to second malignancy. The other 3 were worried about a future risk of infertility.

\section{Conclusions}

Our retrospective study was the first study to define the long-time outcome of a large cohort of multifocal PTC patients less than 20 years old who were treated in China. We hope to investigate the performance of surgery-based multidisciplinary therapy for the multifocal pediatric PTC in the real-world treatment pattern. It is true that most pediatric patients suffered from the more severe stage of disease at the time of diagnosis and most of them need TT and RAI. However, our retrospective results had satisfactory DFS and OS, which opens the possibility of a more conservative treatment strategy that can minimize the side effects in selected children and adolescent patients. The limitation of the study is the relatively short followup time and limited number of cases. The pediatric patients who underwent treatment may have recurrence 20 years later and may have long term complications (secondary malignancy with $\mathrm{RAI}$ ) which may not develop for a decade or more. Unfortunately, short-term follow-up cannot be used to make long-term recommendations. Consequently, these findings should not be overstated. Further research is required to confirm our results.

\section{Acknowledgments}

Funding: This study was supported by the Shanghai Municipal Commission of Health and Family Planning Project (No. 20164Y0214).

\section{Footnote}

Conflicts of Interest: The authors have no conflicts of interest to declare.

Ethical Statement: The authors are accountable for all aspects of the work in ensuring that questions related to the accuracy or integrity of any part of the work are appropriately investigated and resolved. The protocol was approved by the Ethics Committee of Fudan University Shanghai Cancer Center (No. 050432-4-1212B).

\section{References}

1. Chen $\mathrm{W}$, Zheng R, Baade PD, et al. Cancer statistics in China, 2015. CA Cancer J Clin 2016;66:115-32.

2. Siegel RL, Miller KD, Jemal A. Cancer statistics, 2018. CA Cancer J Clin 2018;68:7-30.

3. Josefson J, Zimmerman D. Thyroid nodules and cancers in children. Pediatr Endocrinol Rev 2008;6:14-23.

4. Haugen BR, Alexander EK, Bible KC, et al. 2015 American Thyroid Association Management Guidelines for Adult Patients with Thyroid Nodules and Differentiated Thyroid Cancer: The American Thyroid Association Guidelines Task Force on Thyroid Nodules and Differentiated Thyroid Cancer. Thyroid 2016;26:1-133.

5. Demidchik YE, Demidchik EP, Reiners C, et al. Comprehensive clinical assessment of 740 cases of surgically treated thyroid cancer in children of Belarus. Ann Surg 2006;243:525-32.

6. Handkiewicz-Junak D, Wloch J, Roskosz J, et al. Total thyroidectomy and adjuvant radioiodine treatment independently decrease locoregional recurrence risk in childhood and adolescent differentiated thyroid cancer. J Nucl Med 2007;48:879-88.

7. Hay ID, Gonzalez-Losada T, Reinalda MS, et al. Longterm outcome in 215 children and adolescents with 
papillary thyroid cancer treated during 1940 through 2008. World J Surg 2010;34:1192-202.

8. Hogan AR, Zhuge Y, Perez EA, et al. Pediatric thyroid carcinoma: incidence and outcomes in 1753 patients. J Surg Res 2009;156:167-72.

9. Sosa JA, Tuggle CT, Wang TS, et al. Clinical and economic outcomes of thyroid and parathyroid surgery in children. J Clin Endocrinol Metab 2008;93:3058-65.

10. Lloyd RV, Osamura RY, Klöppel G, et al. WHO classification of tumours: pathology and genetics of tumours of endocrine organs. 4th ed. Lyon: IARC Press, 2017.

11. Kim KJ, Kim SM, Lee YS, et al. Prognostic significance of tumor multifocality in papillary thyroid carcinoma and its relationship with primary tumor size: a retrospective study of 2,309 consecutive patients. Ann Surg Oncol 2015;22:125-31.

12. Zhang W, Jiao D, Liu B, et al. Analysis of Risk Factors Contributing to Recurrence of Papillary Thyroid Carcinoma in Chinese Patients Who Underwent Total Thyroidectomy. Med Sci Monit 2016;22:1274-9.

13. Leboulleux S, Rubino C, Baudin E, et al. Prognostic factors for persistent or recurrent disease of papillary thyroid carcinoma with neck lymph node metastases and/ or tumor extension beyond the thyroid capsule at initial diagnosis. J Clin Endocrinol Metab 2005;90:5723-9.

14. Grogan RH, Kaplan SP, Cao H, et al. A study of recurrence and death from papillary thyroid cancer with 27 years of median follow-up. Surgery 2013;154:1436-46; discussion 1446-7.

15. Vasileiadis I, Karakostas E, Charitoudis G, et al. Papillary thyroid microcarcinoma: clinicopathological characteristics and implications for treatment in 276 patients. Eur J Clin Invest 2012;42:657-64.

16. Pyo JS, Sohn JH, Kang G. Detection of Tumor Multifocality Is Important for Prediction of Tumor Recurrence in Papillary Thyroid Microcarcinoma: A

Cite this article as: Chen J, Huang N, Ji Q, Wang Y, Zhu Y, Li D. Multifocal papillary thyroid cancer in children and adolescents: 12-year experience in a single center. Gland Surg 2019;8(5):507-515. doi: 10.21037/gs.2019.09.03
Retrospective Study and Meta-Analysis. J Pathol Transl Med 2016;50:278-86.

17. Wang F, Yu X, Shen X, et al. The Prognostic Value of Tumor Multifocality in Clinical Outcomes of Papillary Thyroid Cancer. J Clin Endocrinol Metab 2017;102:3241-50.

18. Feinmesser R, Lubin E, Segal K, et al. Carcinoma of the thyroid in children--a review. J Pediatr Endocrinol Metab 1997;10:561-8.

19. Vaisman F, Corbo R, Vaisman M. Thyroid carcinoma in children and adolescents-systematic review of the literature. J Thyroid Res 2011;2011:845362.

20. Francis GL, Waguespack SG, Bauer AJ, et al. Management Guidelines for Children with Thyroid Nodules and Differentiated Thyroid Cancer. Thyroid 2015;25:716-59.

21. Jarzab B, Handkiewicz Junak D, Wloch J, et al. Multivariate analysis of prognostic factors for differentiated thyroid carcinoma in children. Eur J Nucl Med 2000;27:833-41.

22. Welch Dinauer CA, Tuttle RM, Robie DK, et al. Clinical features associated with metastasis and recurrence of differentiated thyroid cancer in children, adolescents and young adults. Clin Endocrinol (Oxf) 1998;49:619-28.

23. Grigsby PW, Gal-or A, Michalski JM, et al. Childhood and adolescent thyroid carcinoma. Cancer 2002;95:724-9.

24. Seidlin SM, Marinelli LD, Oshry E. Radioactive iodine therapy; effect on functioning metastases of adenocarcinoma of the thyroid. J Am Med Assoc 1946;132:838-47.

25. Newman KD, Black T, Heller G, et al. Differentiated thyroid cancer: determinants of disease progression in patients $<21$ years of age at diagnosis: a report from the Surgical Discipline Committee of the Children's Cancer Group. Ann Surg 1998;227:533-41.

26. Bargren AE, Meyer-Rochow GY, Delbridge LW, et al. Outcomes of surgically managed pediatric thyroid cancer. J Surg Res 2009;156:70-3. 